“C 2018 IEEE. Personal use of this material is permitted. Permission from IEEE must be obtained for all other uses, in any current or future media, including reprinting/republishing this material for advertising or promotional purposes, creating new collective works, for resale or redistribution to servers or lists, or reuse of any copyrighted component of this work in other works." 


\title{
Optimal Cost-Based Cyber Insurance Policy Management for Mobile Services
}

\author{
Dinh Thai Hoang, Dusit Niyato, and Ping Wang \\ School of Computer Science and Engineering, Nanyang Technological University (NTU), Singapore
}

\begin{abstract}
This paper introduces a cyber insurance policy management for the mobile networks in which if a mobile user agrees to purchase an insurance policy from an insurer, the loss of the mobile user, i.e., the insured, will be covered by the insurance policy when the risks happen. To protect mobile users from cyber attacks, the insurer can deploy security protection solutions, e.g., anti-virus software or personal firewall, to the insureds, thereby reducing the risks for mobile users. However, when the solutions are deployed, they will incur a certain cost to the insurer. Therefore, we propose a stochastic optimization based on the reserve state of the insurer and the number of active mobile users to determine whether the protection solutions should be deployed or not to maximize the revenue for the insurer. The performance evaluation reveals that the optimal policy can achieve significantly higher revenue than those of baseline schemes for the insurer. Alternatively, the coalitional game is studied to share the reward among the insurers, and we show that the insurers can gain higher individual rewards through the cooperation.
\end{abstract}

Index Terms-Cyber insurance, cyber security, risk management, MDP, coalitional game, wireless attack.

\section{INTRODUCTION}

Cyber security is a paramount issue in wireless communications and mobile networking. Various types of attacks and risks arise in wireless systems and mobile services including denial-of-service (e.g., jamming attack) and eavesdropping in the physical layer, MAC spoofing and network injection in the MAC layer, IP spoofing and IP hijacking in the network layer, TCP and UDP flooding in the transport layer, and malware and SMTP attacks in the application layer. Although a number of advanced security solutions for wireless systems and mobile networks have been introduced [1], achieving complete security protection is still nearly impossible. Therefore, cyber insurance has emerged as an alternative approach to address and manage cyber risks for mobile networks [2]. In particular, when a mobile user agrees to purchase a cyber insurance contract offered by an insurer, the user will receive the protection from the insurer. Thus, the mobile user's risks can be "transferred" to the insurer, and the insurer can profit from the premium and efficient risk management solutions.

To explore the full potential of cyber risk management, the security protection and cyber insurance should be combined [3]. However, due to the moral-hazard effect, the users may recklessly ignore adopting necessary security protection as the users may rely on the cyber insurance. However, in fact, the studies, e.g., [3], showed that there is a balance between employing proper security protection and obtaining cyber insurance policy. As such, the insurer may provide security protection solutions, e.g., intrusion prevention, to the users which buy its cyber insurance product. The security protection will reduce the chance of successful attacks and consequently curtail the claims paid to the affected users.

This paper studies the aforementioned issue in which an insurer will decide to deploy/not to deploy security protection solutions to the mobile users which subscribe to the cyber insurance policy. The objective is to maximize the revenue for the insurer, and thus the insurer has to manage its reserve (money) efficiently. The reserve dynamic depends on the incoming premium paid by the mobile users, and outgoing claims paid back to the users if attacks happen to them. Additionally, if the insurer decides to deploy a security protection solution, the cost is bonded to the reserve. The insurer has to ensure that there is enough reserve for the payment. Otherwise, it has to borrow from an external institution which incurs a certain cost. On the contrary, if the reserve is high, the insurer can invest and receive a certain return. To optimally manage the reserve, we introduce an optimization based on a Markov decision process (MDP) to decide the action to deploy the security protection or not. Moreover, we consider a scenario in which multiple insurers can cooperate to share the reserve, cost, and return of the investment. The solution concept from the coalitional game is applied to share the reward. The numerical results show that the optimization and cooperation yield clear benefits to the insurers.

\section{RELATED WORK}

In [3], a security-as-a-service framework with cyber insurance policies was introduced to allocate security services and manage risks simultaneously for cloud customers. The main objective of this framework is to mitigate the risks, while minimizing the total cost from purchasing security services and cyber insurance policy for the cloud customers. In [4], the authors introduced a cost-aware hierarchical cyber incident analytic framework to help enterprises reduce the cost of cyber insurance without lowing down the level of digital security guarantees. The idea of this paper is to combine business tools with big data techniques to create a classified hierarchy that can distinguish and identify risks and their solutions and costs.

In [5], the authors adopted an insurance model to improve spectrum efficiency in cognitive radio networks (CRNs). In this model, the primary users (PUs) are spectrum sellers (insurers), while the secondary users (SUs) are buyers (insureds). 
Given the insurance policies offered by PUs, the SUs have to decide to simply purchase a channel or meanwhile sign an insurance contract with the PUs to cover the potential accidents, e.g., jamming attacks. Simulations results demonstrated that the utilities of SUs under low risk and high risk will be improved approximately $4.6 \%$ and $23.5 \%$, respectively. Extended from [5], the authors in [6] introduced an idea of using a joint insurance pool established by the PUs to protect themselves against accidental and unpredictable loss, or provide a temporary respite from stagnation. It was shown that partial absorption of the large losses can be achieved due to enhanced cooperation level among PUs.

In [7], an economic model based on cyber insurance was proposed to maximize revenue for plug-in electric vehicles (PEVs) in vehicle-to-grid (V2G) systems. In V2G networks, V2G system information is not always available, and thus the PEVs may be unable to achieve the best charging/discharging performance. Therefore, an insurance policy together with an optimization algorithm was proposed to find optimal decisions for the PEVs in charging and purchasing insurance. Simulation results reveal that cyber insurance is an efficient approach in dealing with cyber risks and maximizing revenue for the PEVs. In [8], the authors introduced a bi-level game-theoretic framework for studying an application of cyber insurance to computer networks. This framework provides an integrative view of the cyber insurance and enables a systematic design of incentive compatible and attack-aware insurance policy.

To the best of our knowledge, this paper is the first which proposes a novel software security-bundled cyber insurance model to protect mobile users from cyber attacks. Moreover, unlike the aforementioned and other works in the literature, we introduce a new idea of using the reserve obtained by premium for other business activities to invest and earn an extra profit. We also develop an MDP framework to find an optimal insurance strategy for the insurer.

\section{System ModeL}

We consider a cyber insurer selling an insurance policy to a set of mobile users in a wireless network (Fig. 1). The user arrival rate is $\lambda$ per minute, and the active user remains in the network for $\mu$ minutes on average. The maximum number of active users in the network is denoted by $N$. The users in the network are vulnerable to cyber attacks from attackers. A user buys the insurance policy from the insurer by paying a premium denoted by $p$ monetary units (MUs) per time period. Hence, the user is the insured. If the attack happens to the user, the insurer will pay a fixed claim with the amount of $c$ MUs to the attacked user. Intrusion detection can be employed to monitor and detect such attacks [9].

The insurer may decide to offer a protection software solution, e.g., malware scan, to the users which buy the insurance policy. Without the protection, the probability that the attacker successfully attacks a user is denoted by $\gamma_{\mathrm{no}}$, and it is $\gamma_{\mathrm{pr}}$ with the protection solution in which $\gamma_{\mathrm{no}}>\gamma_{\mathrm{pr}}$. If the insurer offers the protection solution, it will cost $s$ MUs. The insurer

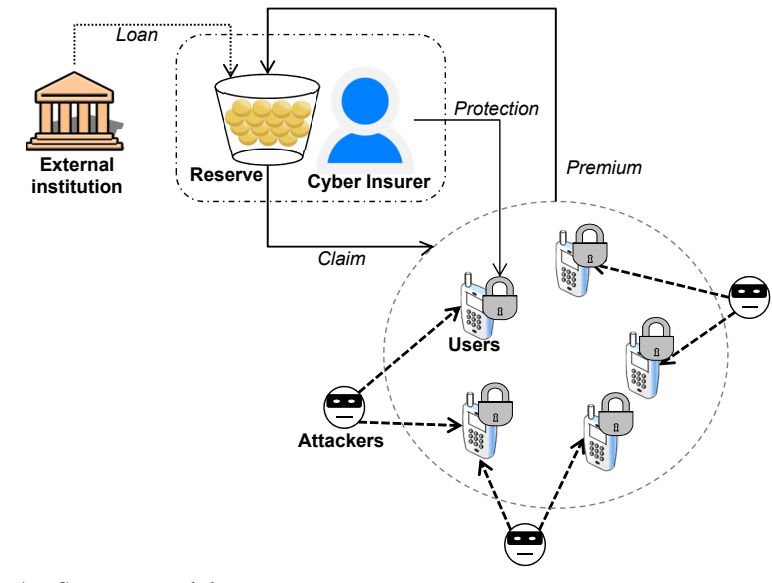

Fig. 1. System model.

has a (cash) reserve for accumulating the premium from the users and paying claims and covering the protection solution. If the reserve is insufficient to pay the claim, the insurer has to borrow money from external institution which costs the insurer $b>1$ MUs per one MU that the insurer borrows. On the contrary, the insurer has a long-term investment plan in which if the reserve is higher than a certain threshold $R$, the insurer will invest the excess reserve which yields $v>1$ MUs per one MU that the insurer invests.

\section{OPTIMIZATION FORMULATION}

We formulate the decision making of the insurer as a Markov decision process (MDP) model. The state space is defined as follows:

$$
\Omega=\{(\mathscr{R}, \mathscr{N}) ; \mathscr{R} \in\{0,1, \ldots, R\}, \mathscr{N} \in\{0,1, \ldots, N\}\},
$$

where $\mathscr{R}$ is the reserve state which can take a value between zero and the maximum reserve threshold $R$, and $\mathscr{N}$ is the number of active users which can take a value between zero and the maximum number of users $N$. The action space is defined as follows $\Delta=\{0,1\}$, in which 0 and 1 represent that the insurer does not and does deploy the protection to the active user, respectively.

Let $r$ and $r^{\prime}$ be the current and next reserve states, respectively. If the security protection is not deployed, the reserve state change can be expressed as follows:

$$
r^{\prime}=r-n^{\dagger} c+n p
$$

The reserve increases by the premium $p$ collected from $n$ active users and it decreases by the claim $c$ paid to the $n^{\dagger}$ active users who have been attacked. However, if the protection is deployed, the reserve state change is

$$
r^{\prime}=r-n^{\dagger} c-s+n p,
$$

where $s$ is the cost of deploying the protection. Here, if $r^{\prime}<0$, the insurer has to borrow money which will cost $-b r^{\prime}$. On the contrary, if $r^{\prime}>R$, i.e., the reserve is more than the threshold 
$R$, the insurer can invest excess reserve and gain $+v\left(r^{\prime}-R\right)$. The reserve can decrease if the attack happens. The probability that the claim amount $n^{\dagger} c$ has to be paid is obtained by

$$
\theta\left(n^{\dagger} c\right)=\left(\begin{array}{c}
N c \\
n^{\dagger} c
\end{array}\right) \gamma^{n^{\dagger} c}(1-\gamma)^{\left(N-n^{\dagger}\right) c}
$$

for $n^{\dagger} \in\{0,1, \ldots, N\}$, where $\gamma=\gamma_{\text {no }}$ if the protection is deployed and $\gamma=\gamma_{\mathrm{pr}}$ if the protection is not deployed.

In the following, we derive the transition probability matrix of the MDP. We first consider the number of active users, which can thus be modeled as a Markov chain [10]. The corresponding transition matrix is expressed as follows:

$$
\mathbf{Q}=\left[\begin{array}{cccc}
-\lambda & \lambda & & \\
\mu & -\lambda-\mu & \ddots & \ddots \\
& \ddots & \lambda & \lambda \\
& (N-1) \mu & -\lambda-(N-1) \mu & \lambda \mu
\end{array}\right],
$$

where each row corresponds to the number of active users $n$. To convert $\mathbf{Q}$ to the transition probability matrix, we apply the uniformization method as follows:

$$
\mathbf{N}=\frac{\mathbf{Q}}{\kappa}+\mathbf{I}, \quad \text { for } \quad \kappa \geq \min _{y}\left(\left|[\mathbf{Q}]_{y, y}\right|\right) .
$$

$[\mathbf{Q}]_{y, y}$ denotes the diagonal element at row $y$ and column $y$ of matrix Q. In other words, $\kappa$ is greater than or equal to the absolute value of the minimum diagonal element of $\mathbf{Q}$.

Next, we consider the insurer's reserve state. The corresponding transition probability $\mathbf{P}^{(a)}$ is expressed as in (7) for action $a \in \Delta$. Each row of matrix $\mathbf{P}^{(a)}$ represents the reserve state. The reserve state can increase and decrease depending on the events happening. $\otimes$ is the Kronecker product in which the reserve state transition is combined with the transition of number of active users, i.e., N. Here, $\beta^{\prime}(a)$ denotes the maximum decrease while $\beta^{\prime \prime}(a)$ denotes the maximum increase of the reserve state. We have $\beta^{\prime}(a)=N c$ and $\beta^{\prime \prime}(a)=N p$ when action $a=0$ is taken, i.e., no protection is deployed. On the contrary, we have $\beta^{\prime}(a)=N c+s$ and $\beta^{\prime \prime}(a)=N p-s$ when action $a=1$ is taken, where $s$ is the amount of reserve used to pay for deploying the protection.

Matrix $\mathbf{R}_{r, r^{\prime}}^{(a)}$ contains the transition probability of number of active users when the reserve state changes from $r$ to $r^{\prime}$. It is obtained by combining with the transition matrix of the number of active users, i.e., $\mathbf{R}_{r, r^{\prime}}^{(a)}=\tilde{\mathbf{R}}_{r, r^{\prime}}^{(a)} \times \mathbf{N}$. Let $\left[\tilde{\mathbf{R}}_{r, r^{\prime}}^{(a)}\right]_{n, n}$ denote the diagonal element of matrix $\tilde{\mathbf{R}}_{r, r^{\prime}}^{(a)}$ at row $n$ and column $n$. It is the probability that the reserve state changes from $r$ to $r^{\prime}$ when the number of active users is $n$.

The immediate reward function of the MDP is defined as follows: $R((r, n), a)=$

$$
\begin{cases}\sum_{r^{\prime}=-N c+r}^{-1}\left(r^{\prime} b\right) \overrightarrow{\mathbf{e}}^{\top} \tilde{\mathbf{R}}_{r, r^{\prime}}^{(a)} \overrightarrow{\mathbf{1}}, & \text { if } r^{\prime}<0 \text { and } a=0, \\ \sum_{r^{\prime}=R+1}^{r+N p}\left(r^{\prime} v\right) \overrightarrow{\mathbf{e}}^{\top} \tilde{\mathbf{R}}_{r, r^{\prime}}^{(a)} \overrightarrow{\mathbf{1}}, & \text { if } r^{\prime}>R \text { and } a=0, \\ \sum_{r^{\prime}=-N c-s+r}^{-1}\left(r^{\prime} b\right) \overrightarrow{\mathbf{e}}^{\top} \tilde{\mathbf{R}}_{r, r^{\prime}}^{(a)} \overrightarrow{\mathbf{1}}, & \text { if } r^{\prime}<0 \text { and } a=1, \\ \sum_{r^{\prime}=R+1}^{r+N p-s}\left(r^{\prime} v\right) \overrightarrow{\mathbf{e}}^{\top} \tilde{\mathbf{R}}_{r, r^{\prime}}^{(a)}, & \text { if } r^{\prime}>R \text { and } a=1, \\ 0, & \text { otherwise }\end{cases}
$$

where $\overrightarrow{\mathbf{e}}^{\top}=\left[\begin{array}{llll}0 & 1 & \cdots & N\end{array}\right]$ and $\overrightarrow{\mathbf{1}}$ is a vector of ones. Here, when $r<0$, it incurs the borrowing cost to the insurer, and hence $b$ is applied to every MU of insufficient reserve. Note that in the first and the third conditions, the summation is over the insufficient reserve to be paid for the claim, and hence the sign of $r^{\prime}$ is negative.

The policy of the MDP is the mapping from state $(r, n)$ to action $a$. To obtain the optimal policy, we transform the MDP into an equivalent linear programming (LP) problem. Let $\phi((r, n), a)$ denote the steady state probability that at state $(r, n)$, action $a$ is taken. The equivalent linear programming can be expressed as follows:

$$
\begin{aligned}
& \max _{\phi((r, n), a)} \sum_{(r, n) \in \Omega} \sum_{a \in \Delta} R((r, n), a) \phi((r, n), a), \\
& \text { s.t. } \sum_{a \in \Delta} \phi\left(\left(r^{\prime}, n^{\prime}\right), a\right)= \\
& \sum_{(r, n) \in \Omega} \sum_{a \in \Delta} P\left(\left(r^{\prime}, n^{\prime}\right) \mid(r, n), a\right) \phi((r, n), a), \\
& \sum_{(r, n) \in \Omega} \sum_{a \in \Delta} \phi((r, n), a)=1, \phi((r, n), a) \geq 0,
\end{aligned}
$$

where $P\left(\left(r^{\prime}, n^{\prime}\right) \mid(r, n), a\right)$ is the probability that the current state is $(r, n)$ and the next state is $\left(r^{\prime}, n^{\prime}\right)$ when action $a$ is taken. This probability is the element of matrix $\mathbf{P}^{(a)}$ defined in (7). By solving the aforementioned optimization problem (e.g., using MATLAB toolbox), we can find the optimal policy and derive the average reward of the insurer.

\section{INSURER COOPERATION}

We consider the case that more than one insurer can cooperate to aggregate their users and share a single pool of reserve. The cost and return of investment are shared among the cooperative insurers. The reward dividing is studied using a tool from the coalitional game theory. Let $\mathcal{I}$ denote a set of cooperative insurers. For all the cooperative insurers, they have and share one reserve. The number of active users from all insurers are treated as a single group. Therefore, the state space of the collective insurer, i.e., a group of cooperative insurers, adopted from (1) is denoted by

$\Omega_{\mathcal{I}}=\left\{\left(\mathscr{R}_{\mathcal{I}}, \mathscr{N}_{\mathcal{I}}\right) ; \mathscr{R}_{\mathcal{I}} \in\{0,1, \ldots, R\}, \mathscr{N}_{\mathcal{I}} \in\left\{0,1, \ldots, N_{\mathcal{I}}\right\}\right\}$

where $N_{\mathcal{I}}=\sum_{i \in \mathcal{I}} N_{i}$ and $N_{i}$ is the maximum number of active users of insurer $i$. Since all the cooperative insurers support all users, the transition matrix $Q$ in (5) becomes

$$
\mathbf{Q}_{\mathcal{I}}=\left[\begin{array}{cccc}
-\lambda_{\mathcal{I}} & \lambda_{\mathcal{I}} & & \\
\mu & -\lambda_{\mathcal{I}}-\mu & \lambda_{\mathcal{I}} & \\
& \ddots & \ddots & \ddots \\
& \left(N_{\mathcal{I}}-1\right) \mu & -\lambda_{\mathcal{I}}-\left(N_{\mathcal{I}}-1\right) \mu & \lambda_{\mathcal{I}} \\
& & N_{\mathcal{I}} \mu & -N_{\mathcal{I}} \mu
\end{array}\right]
$$

where $\lambda_{\mathcal{I}}=\sum_{i \in \mathcal{I}} \lambda_{i}$ in which $\lambda_{i}$ is the user arrival rate at insurer $i$. We then follow the derivations of transition probability matrix, reward function, and optimal policy, presented 


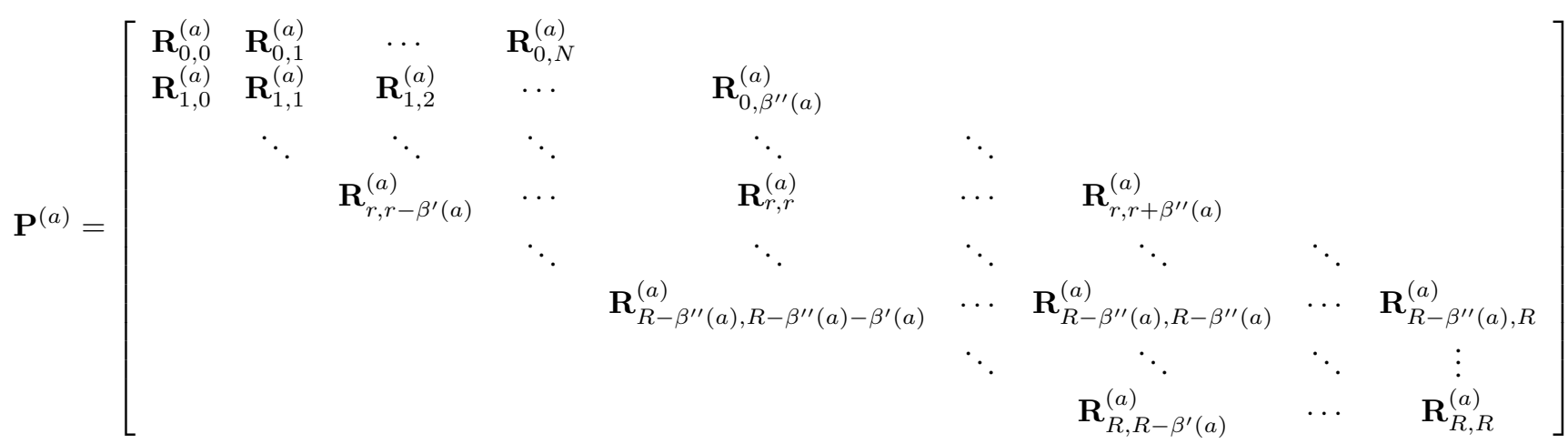

in Section IV. With the cooperation, the reward is aggregated for all cooperative insurers and it is denoted by $W_{\mathcal{I}}$. This aggregated reward has to be divided among all the insurers. In this paper, we apply the Shapley value in which the individual reward of cooperative insurer $i$ is given by

$$
W_{i}=\sum_{\mathcal{S} \subseteq \mathcal{I} \backslash\{i\}} \frac{|\mathcal{S}| !(|\mathcal{I}|-|\mathcal{S}|-1) !}{|\mathcal{I}| !}\left(W_{\mathcal{S} \cup\{i\}}-W_{\mathcal{S}}\right),
$$

where $\mathcal{S}$ is any subset of all cooperative insurers $\mathcal{I}$, and $W_{\mathcal{S}}$ is the aggregated reward of a subset of cooperative insurers.

\section{PERformance Evaluation}

\section{A. Parameter Setting}

The maximum number of users in the network is set to 10 . The arrival rate is 6 users per hour, and the connection holding time is 20 minutes. The premium is 1 monetary unit (MU), and the claim is 5 MUs. The maximum reserve capacity is 300 MUs. The cost of borrowing money because of insufficient reserve is 1.07 MUs and the return of investment from extra reserve is 1.03 MUs. The attack probability is 0.2 , and the protection success probability is 0.75 .

\section{B. Numerical Results}
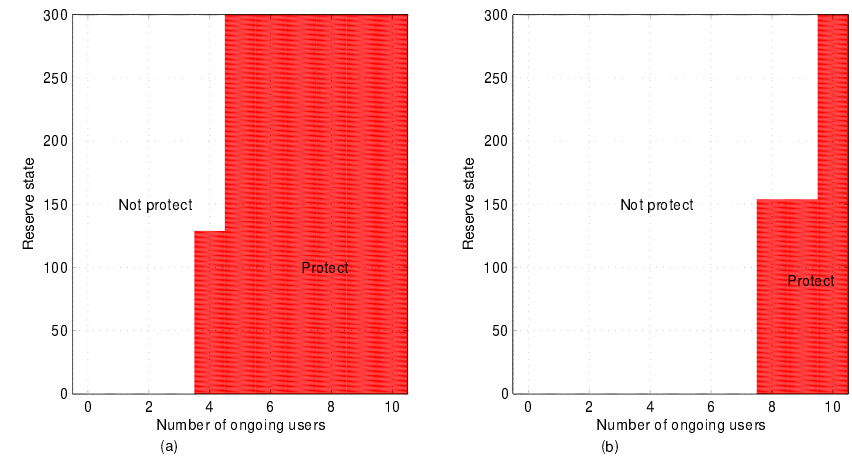

Fig. 2. Policies of the insurer for (a) high success protection probability $(0.5)$ and (b) low success protection probability $(0.25)$.

Figs. 2(a) and (b) show the actions taken by the insurer to deploy the security protection solution to the active users or not. The actions are shown according to the reserve state and the number of active users. Clearly, the insurer will deploy the protection only when the number of active users is large enough. This is due to the fact that the deployment yields higher gain than the cost. Furthermore, the insurer is more likely to deploy the protection when the reserve state is small. This is to avoid the risk of having insufficient reserve which can cost the insurer more than not deploying the protection. When the effectiveness of the protection becomes smaller, e.g., due to adaptive attacks, the insurer is less likely to deploy the protection, i.e., Fig. 2(a) versus Fig. 2(b).

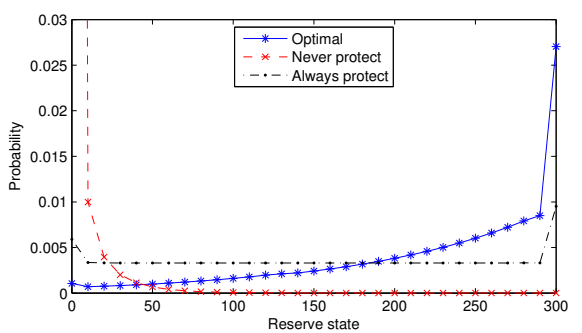

Fig. 3. Distribution of reserve state.

Fig. 3 shows the distribution of reserve state of three policies, i.e., optimal, never protect, and always protect. The "never protect" policy results in the reserve to be empty with high probability, costing the insurer considerably. Alternatively, the "always protect" policy results in more uniform probability of reserve state. On the contrary, the optimal policy leads to the insurer having more reserve than the other two policies. The reward of the optimal policy is thus higher. These results suggest that if the security protection is appropriately applied, the reward of the insurer can be maximized.

Clearly, when the protection success probability increases, the insurer will be more likely to deploy the security protection (Fig. 4(a)). Accordingly, the reward of the insurer increases as the protection success probability increases. Nevertheless, the impact from the protection success probability subsides when the attack probability decreases. This is due to the fact that when the attack happens more frequently, the insurer can deploy the protection to reduce the amount of claim to be paid. 


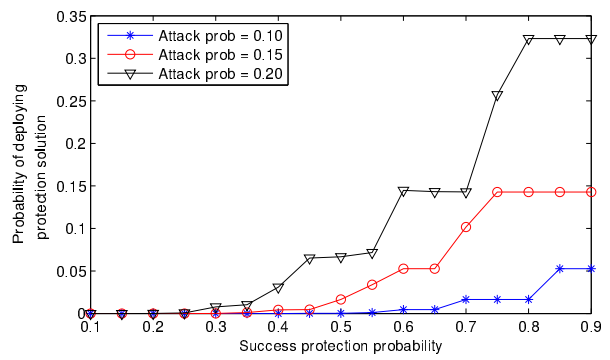

(a)

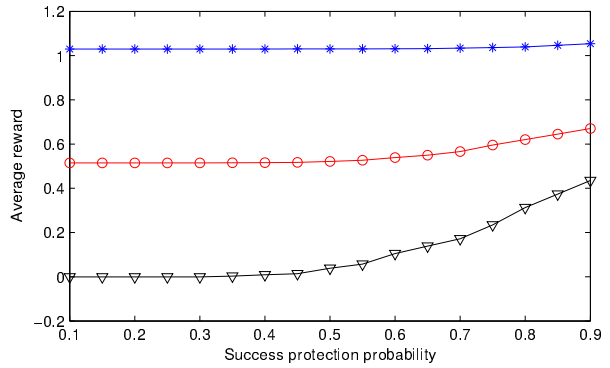

(b)

Fig. 4. (a) The probability of deploying protection action and (b) the average reward of the insurer when the protection success probability is varied.

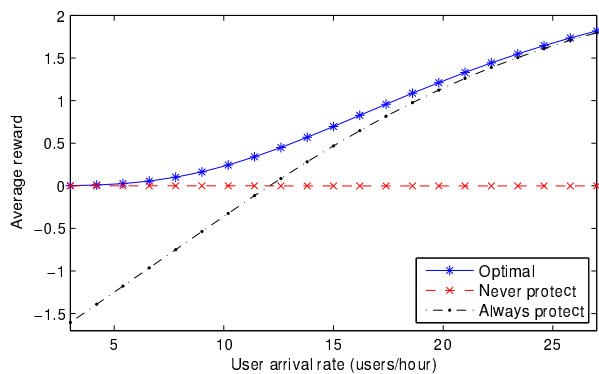

Fig. 5. Average reward when the user arrival rate is varied.

In Fig. 5, when the user arrival rate increases, the average reward increases as the insurer can generate more reward from selling the insurance policy. Again, the proposed optimal policy yields the highest reward. Next, we consider two insurers with and without cooperation. Their average individual rewards are shown in Fig. 6 in which the user arrival rate of insurer 2 is varied while that of insurer 1 is fixed. With cooperation, two insurers share their reserves, and their revenue and cost are also divided, i.e., using the Shapley value. There are two important observations in Fig. 6. First, when two insurers cooperate, they achieve the average individual reward larger than that without the cooperation. Second, with cooperation even when insurer 1 has fixed arrival rate of users, it still gains higher reward when the user arrival rate of insurer 2 increases. Evidently, there is a benefit of cooperation among insurers.

\section{SUMmary}

In this paper, we have considered a cyber insurance scheme for mobile users. The MDP model has been proposed to

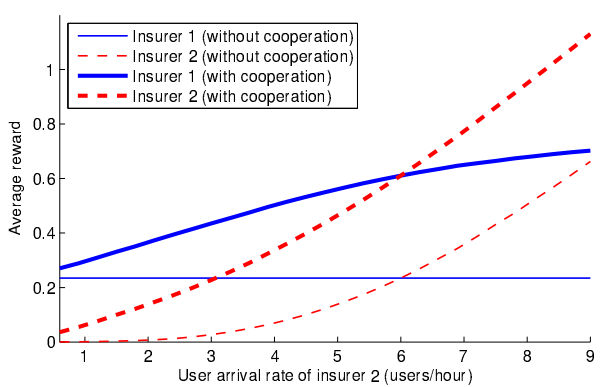

Fig. 6. Average individual reward of two insurers with/without cooperation.

achieve the objective in which the insurer takes the reserve state and the number of active users to derive an optimal policy. We have also considered the situation that multiple insurers can cooperate to share their reserve, cost, and reward. The cooperation can help the cooperative insurers achieve higher individual reward than that without the cooperation. For the future work, we will consider coalition formation among the insurers.

\section{ACKNOWLEDGEMENTS}

This work was supported in part by Singapore MOE Tier 1 (RG 18/13 and RG 33/16) and MOE Tier 2 (MOE2014-T2-2015 ARC4/15 and MOE2013-T2-2-070 ARC16/14).

\section{REFERENCES}

[1] Y. Zou, J. Zhu, X. Wang and L. Hanzo, "A survey on wireless security: Technical challenges, recent advances, and future trends," in Proceedings of the IEEE, vol. 104, no. 9, pp. 1727-1765, Sept. 2016.

[2] R. Pal, L. Golubchik, K. Psounis, and P. Hui, "Will cyber-insurance improve network security? A market analysis," in IEEE Conference on Computer Communications, pp. 235-243, Toronto, Canada, May 2014.

[3] S. Chaisiri, R. Ko, and D. Niyato, "A joint optimization approach to security-as-a-service allocation and cyber insurance management," in Proceedings of IEEE International Conference on Trust, Security and Privacy in Computing and Communications (TrustCom), Helsinki, Finland, 20-22 August, 2015.

[4] K. Gai, M. Qiu, and S. A. Elnagdy, "A novel secure big data cyber incident analytics framework for cloud-based cybersecurity insurance," in International Conference on Big Data Security on Cloud, pp. 171176, New York, US, Apr. 2016.

[5] H. Jin, G. Sun, X. Wang, Q. Zhang, "Spectrum trading with insurance in cognitive radio networks," in INFOCOM, pp. 2041-2049, Orlando, FL, USA, Mar. 2012.

[6] D. Horvath, V. Gazda, and J. Gazda, "Agent-based modeling of the cooperative spectrum management with insurance in cognitive radio networks," EURASIP Journal on Wireless Communications and Networking, no. 1, pp. 1-14, Dec. 2013.

[7] D. T. Hoang, P. Wang, D. Niyato, and E. Hossain, "Charging and discharging of plug-in electric vehicles (PEVs) in vehicle-to-grid (V2G) systems: A cyber insurance-based model," IEEE Access, vol. 5, pp. 732-754, Jan. 2017.

[8] R. Zhang, Q. Zhu, and Y. A. Hayel, "Bi-level game approach to attack-aware cyber insurance of computer networks," IEEE Journal on Selected Areas in Communications, Feb 2017.

[9] N. Marchang, R. Datta and S. K. Das, "A novel approach for efficient usage of intrusion detection system in mobile ad hoc networks," in IEEE Transactions on Vehicular Technology, vol. 66, no. 2, pp. 16841695, Feb. 2017.

[10] Y. Fang and Y. Zhang, "Call admission control schemes and performance analysis in wireless mobile networks," IEEE Transactions on Vehicular Technology, vol. 51, no. 2, pp. 371-382, Mar 2002. 